\title{
A new method to identify flux ropes in space plasmas
}

\author{
Shiyong Huang ${ }^{1, *}$, Pufan Zhao ${ }^{1}$, Jiansen $\mathrm{He}^{2}$, Zhigang Yuan ${ }^{1}$, Meng Zhou ${ }^{3}$, Huishan $\mathrm{Fu}^{4}$, Xiaohua Deng ${ }^{3}$, Ye Pang ${ }^{3}$, \\ Dedong Wang ${ }^{1}$, Xiongdong $\mathrm{Yu}^{1}$, Haimeng $\mathrm{Li}^{4}$, Roy Torbert ${ }^{5}$, and James Burch \\ ${ }^{1}$ School of Electronic Information, Wuhan University, Wuhan, China \\ ${ }^{2}$ School of Earth and Space Sciences, Peking University, Beijing, China \\ ${ }^{3}$ Institute of Space Science and Technology, Nanchang University, Nanchang, China \\ ${ }^{4}$ School of Space and Environment, Beihang University, Beijing, China \\ ${ }^{5}$ University of New Hampshire, Durham, New Hampshire, USA \\ ${ }^{6}$ Southwest Research Institute, San Antonio TX, USA \\ * Invited contribution by Shiyong Huang, recipient of the EGU Planetary and Solar System Sciences \\ Division Outstanding Early Career Scientists Award 2016.
}

Correspondence: Shiyong Huang (shiyonghuang@msn.com)

Received: 4 May 2018 - Discussion started: 14 May 2018

Revised: 8 September 2018 - Accepted: 11 September 2018 - Published: 1 October 2018

\begin{abstract}
Flux ropes are frequently observed in the space plasmas, such as solar wind, planetary magnetosphere and magnetosheath etc., and play an important role in the reconnection process and mass and flux transportation. One usually uses bipolar signature and strong core field to identify the flux ropes. We propose here one new method to identify flux ropes based on the correlations between the variables of the data from in situ spacecraft observations and the "target function to be correlated" (TFC) from the ideal flux rope model. Through comparing the correlation coefficients of different variables at different times and scales, and performing weighted-average techniques, this method can derive the scales and locations of the flux ropes. We compare it with other methods and also discuss the limitation of our method.
\end{abstract}

\section{Introduction}

Magnetic flux ropes, as one universal structure in the space plasma, are formed as a helical magnetic structure with magnetic field lines wrapping and rotating around a central axis (e.g., Hughes and Sibeck, 1987; Slavin et al., 2003; Zong et al., 2004; Zhang et al., 2010). It is generally believed that flux ropes can be generated by magnetic reconnection in the eruptive energy processes, such as rapid variations of the reconnection rate at a single $\mathrm{X}$ line (e.g., Nakamura and Scholer, 2000; Wang et al., 2010; Fu et al., 2013) or multiple Xline reconnections (e.g., Lee et al., 1985; Deng et al., 2004).
Flux ropes play important roles in dissipating magnetic energy and controlling the microscale dynamics of magnetic reconnection (e.g., Drake et al., 2006; Daughton et al., 2007; Wang et al., 2016; Fu et al., 2017). These structures have been frequently observed and widely studied recently in the magnetosphere, magnetosheath and solar wind (e.g., Hu and Sonnerup, 2001; Slavin et al., 2003; Zong et al., 2004; Zhang et al., 2010; Huang et al., 2012, 2014a, b, 2015, 2016a, b; Rong et al., 2013). Many works have tried to model flux rope from in situ measurements based on the force-free constantalpha flux rope (e.g., Lepping et al., 1990), the non-force-free model (e.g., Hidalgo et al., 2002), or the Grad-Shafranov equilibrium (e.g., Hu and Sonnerup, 2002).

Flux ropes embedded in current sheet are characterized by the bipolar signature of the normal component of a magnetic field, strong core field in the axis direction and enhancement in magnetic field strength. Therefore, one uses negativepositive (positive-negative) bipolar signatures of the southnorth magnetic field component in the earthward (tailward) flow with an enhancement in the cross-tail component and strength of magnetic field to identify flux ropes in the magnetotail (e.g., Slavin et al., 2003; Huang et al., 2012). At the magnetopause, the bipolar variation is usually along the Sun-Earth direction, and the core field is typically along the dawn-dusk direction (e.g., Zhang et al., 2010). However, flux ropes in the magnetosheath, which has been reported recently by MMS (Magnetospheric Multiscale mission; Huang et al., 2016b), can move in any direction due to the large fluctua- 
tions of the shocked solar wind. This leads to difficultly in identifying the flux ropes there.

Several attempts are made to survey flux ropes in the Earth's magnetotail by eyes based on their signatures, such as bipolar variation of the north-south magnetic field (e.g., Richardson et al., 1987; Slavin et al., 2003). Also, some methods are proposed to automatically, in some degrees, survey flux ropes or flux transfer events (FTEs) via bipolar field deflections (e.g., Kawano and Russell, 1996; Vogt et al., 2010; Jackman et al., 2014; Smith et al., 2016). Karimabadi et al. (2009) have applied a data mining technique (MineTool) to search FTEs using magnetic field and plasma data. Recently, Smith et al. (2017) developed a method to automatically detect cylindrically symmetric force-free flux ropes in the magnetotail only using magnetic field data. That method first locates the significant deflections in the north-south magnetic field component with peaks in the dawn-dusk component or total field. Then, the candidates use minimum variance analysis (MVA) to determine a local coordinate system. Finally, the candidates are fitted by a force-free model to determine whether they belong to flux ropes or not.

For some flux ropes with short duration, the plasma data do not have enough high time resolution or, even worse, are not available. Thus, the identification of flux ropes relies heavily on the magnetic field data. All aforementioned automatic methods are a bit complex, or require plasma data. Therefore, to identify flux rope only using the magnetic field data from a single spacecraft, we propose a new and simple method based on the correlation coefficients between the signal and the ideal model of flux rope to identify flux ropes in space plasmas. The paper will be presented as follows: an introduction of the method in Sect. 2, the test of the method on artificial data from the model in Sect. 3, the applications of the method on the Cluster and MMS data in Sect. 4, and the summary given in Sect. 5 .

\section{Approach}

In this section, we simply introduce our method.

Firstly, we derive the "target function to be correlated" (TFC) from the ideal model of flux rope. Considering the variable and complicated observed flux ropes, we use the ideal non-force-free model of flux rope proposed by Elphic and Russell (1983), named the Elphic and Russell $(E-R)$ model because most of flux ropes with nonnegligible perpendicular currents are not consistent with the force-free model (e.g., Hidalgo et al., 2002; Zong et al., 2004; Zhang et al., 2010; Borg et al., 2012; Huang et al., 2012, 2016b). This model is constructed with an intense core field inside of flux rope, which is shown in Fig. 1. The equation of this model in the cylindrical coordinate ( $Y$ is defined as the axis orientation of flux rope) can be modified as below:

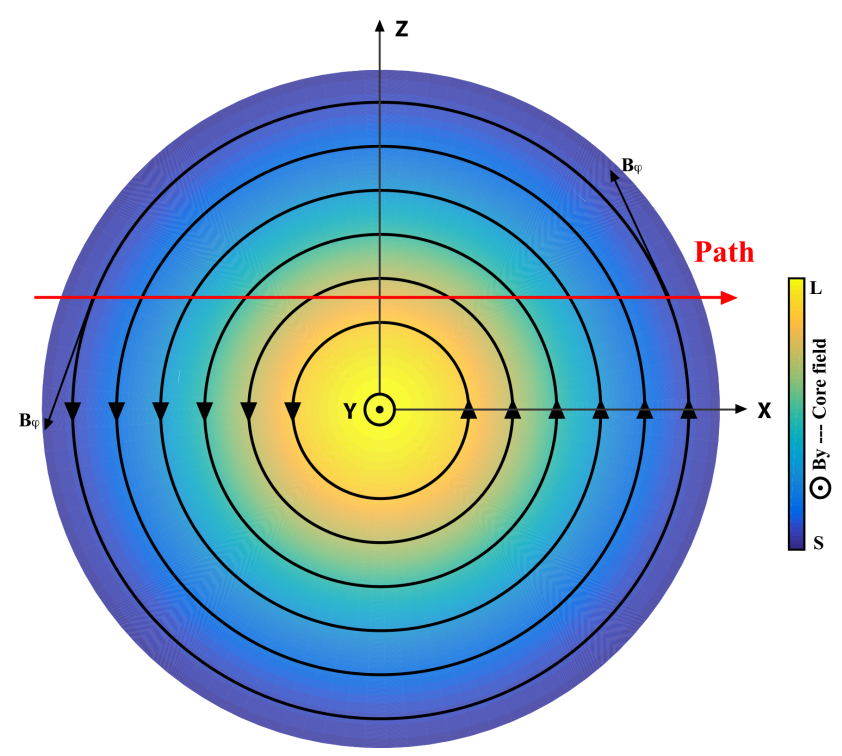

Figure 1. Sketched diagram of the cylindrical flux rope. The flux rope has a right-handed structure. The black circled lines are the magnetic field lines. The red arrow is the projection of spacecraft path. The rectangular coordinate is used in our analyses. $Y$ is the axis orientation of the flux rope, and the $X-Z$ plane is the cross section perpendicular to the axis orientation. The core field is out of plane, and the color represents the relative strength of core field (yellow: large, blue: small).

$$
\left\{\begin{array}{l}
B_{y}=B(r) \cos (\alpha(r)), \\
B_{\varphi}=B(r) \sin (\alpha(r)), \\
B(r)=B_{0} \exp \left(-r^{2} / b^{2}\right),
\end{array}\right.
$$

where $\alpha(r)=\pi / 2\left(1-\exp \left(-r^{2} / a^{2}\right)\right) ; B_{y}$ is the core field component; $B_{0}, a$, and $b$ are the constants; and $r$ is the radial distance to the flux rope center.

Figure 1 shows a sketched diagram of the cylindrical flux rope from the $E-R$ model. For convenience, the rectangular coordinate is used in our analyses (shown in Fig. 1). $Y$ is the axis orientation of the flux rope, and the $X-Z$ plane is the cross section perpendicular to the axis orientation. $X$ can be treated as Sun-Earth orientation, $Y$ is the dawn-dusk orientation, and $Z$ is similar to the south-north orientation in the magnetotail. If one spacecraft crosses the flux rope following the red path in Fig. 1, the $B_{z}$ component will be characterized as bipolar signature, and the $B_{y}$ component and total magnetic field $B_{t}$ have strong peaks.

Figure 2 shows the observations when one virtual spacecraft crosses the ideal flux rope (see spacecraft path in Fig. 1). Here we assume the scale of flux rope as one unit, and 1 unit $^{-1}$ of moving speed of the spacecraft, thus set $a=$ 0.735 units and $b=0.735$ units, $B_{0}=10 \mathrm{nT}$, and use the $B_{z}$ as the bipolar variation component, $B_{y}$ as the core field component, $B_{\mathrm{t}}$ as the total magnetic field. The center of the flux rope is located at $2.5 \mathrm{~s}$. One can see the $B_{z}$ bipolar signature, 

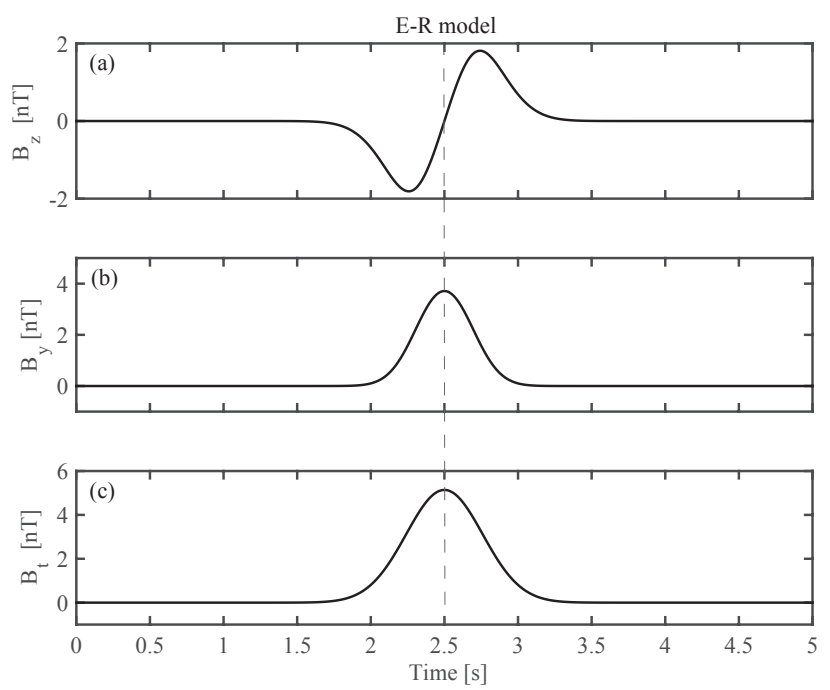

Figure 2. The three variables $B_{z}(\mathbf{a}), B_{y}(\mathbf{b})$ and $B_{\mathrm{t}}$ (c) of the ideal cylindrical flux rope described by the $E-R$ model.

and the peaks of core field and total magnetic field inside the flux rope.

Considering the previous observations, in which the $B_{z}$ component during the crossing of the flux rope usually does not reach zero like that shown in Fig. 2a, we select one part of the ideal flux rope as the TFC which is shown in Fig. 3. The TFC is similar to the sinusoidal function when one performs fast Fourier transform (FFT) analysis. We only used two components $\left(B_{y}\right.$ and $\left.B_{z}\right)$ and magnetic strength $\left(B_{\mathrm{t}}\right)$ as the TFC since only $B_{z}$ and $B_{y}$ components and $B_{\mathrm{t}}$ have very obvious typical features usually from in situ measurements (i.e., $B_{z}$ has bipolar signature, $B_{y}$ is strong core field, and $B_{\mathrm{t}}$ has peak inside flux ropes), and $B_{x}$ component does not have common features from observation viewpoint (e.g., Slavin et al., 2003; Huang et al., 2014a).

Secondly, we calculate the Pearson correlation coefficients between the signal and the TFC at different times and different scales (Hotelling, 1953). Before calculating the correlation coefficients, the amplitude of the TFC will be estimated from the signal. For example, the maximum value of $B_{\mathrm{t}}$ during the time interval is used as the amplitude of $B_{\mathrm{t}}$ in the TFC. The sliding time window is used in the calculation of the correlation coefficients. The calculated results of correlation coefficients are similar to the power spectral densities by FFT that display the power spectral density at different times and different frequencies. The higher the values of the correlation coefficients, the more suitable for the description of the model on the signal.

Thirdly, we compare the correlation coefficients of the bipolar variation component $B_{z}$, core field component $B_{y}$ and total magnetic field $B_{\mathrm{t}}$, and find out the high correlations (larger than the given threshold) at the same time and the same scale. This is due to the fact that the bipolar sig-
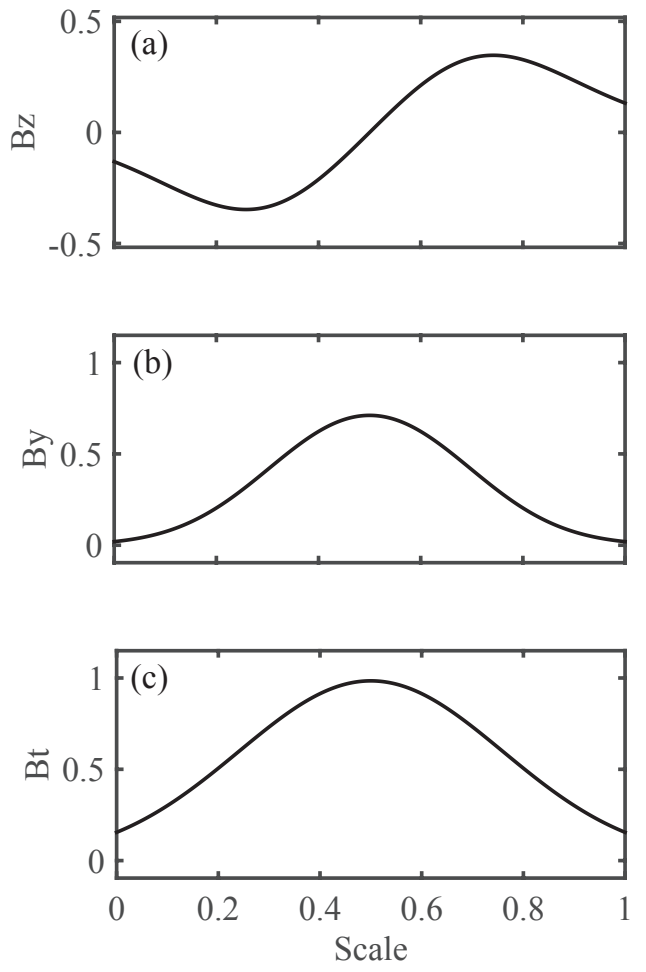

Figure 3. The target-function-to-be-correlated (TFC) derived from $E-R$ model. The amplitudes and scale are dimensionless.

nature in $B_{z}$, enhancements of core field $B_{y}$ and magnetic strength $B_{\mathrm{t}}$ should appear simultaneously with the same duration when one spacecraft crosses the flux ropes.

Fourthly, we infer the location and the scale of the flux ropes based on the weighted average (it will be shown later), and the amplitude from minimum to maximum values of the bipolar variation.

\section{Model test}

One test is performed on the artificial data from $E-R$ model with the random noise. Figure 4 presents the test results. The test artificial data are shown in Fig. $4 \mathrm{a}$ where the noise is $10 \%$ of the amplitude of the flux rope. A series of the calculations are carried on $B_{z}, B_{y}$ and $B_{\mathrm{t}}$ to obtain the correlation coefficients. One should point out that the absolute values of the correlation coefficients of $B_{z}$ and $B_{y}$ are given in Fig. $4 \mathrm{~b}$ and c respectively, because the bipolar structure can be positive-negative or negative-positive variation and the core field can be positive or negative. It can be seen that the correlation coefficients are largest at the scale $\tau$ of 0.61.5 units during the crossing of the flux rope (around time $\sim$ $3.5 \mathrm{~s})$.

We set the threshold as 0.9 to represent the results in Fig. 5 where only the correlation coefficients with $>0.9$ are displayed with black shadows. All correlation coefficients of the 

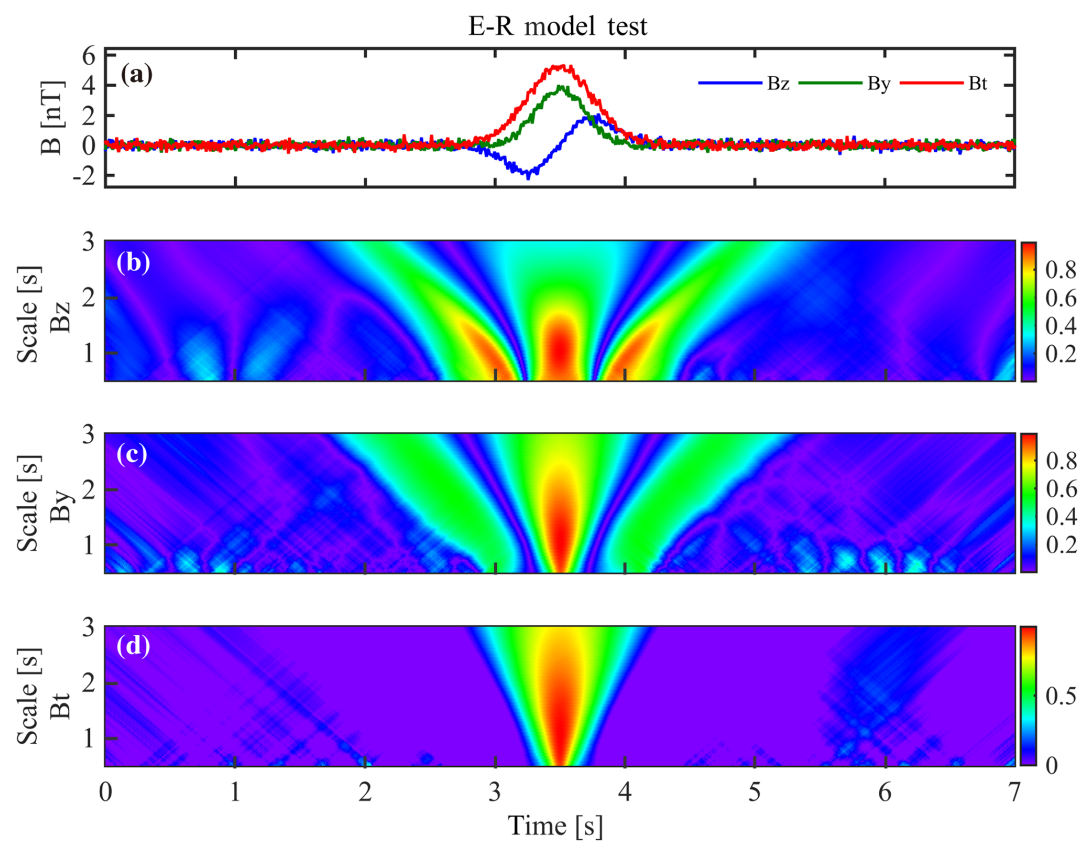

Figure 4. The test results on the $E-R$ model. (a) Three variables $B_{z}, B_{y}$ and $B_{\mathrm{t}}$ from $E-R$ model with $10 \%$ random noise; (b-d) the correlation coefficients between the variables of $B_{z}, B_{y}$ and $B_{\mathrm{t}}$ and the TFC shown in Fig. 3, respectively. The scale on the vertical axes of (b-d) is $\tau$, as mentioned in the text, which can also be seen as a unit.

three variables have peaks at the time $\sim 3.5 \mathrm{~s}$ with the scale $\tau \sim 1$ units. We use the weighted-average technique (shown below) to identify the flux rope and estimate its scale $\tau$.

$\tau=\sum \operatorname{coef}_{i} \times \tau_{i} / \sum \operatorname{coef}_{i}$

where coef $_{i}$ is the correlation coefficient at scale $\tau_{i}$.

Figure $5 \mathrm{e}$ shows the estimated results. The crossing of the flux rope is marked with " 1 " and the duration is its scale, and the center of the flux rope is at the center of the line. In this test, the scale is estimated as 1.039 units, and the location is $3.496 \mathrm{~s}$. The amplitude is estimated to be $4.43 \mathrm{nT}$ from minimum to maximum values of the bipolar variation. Aforementioned sets, one can estimate the error of the scale as $3.9 \%$, i.e., $(1.039-1.0) / 1.0=3.9 \%$. Therefore, our method can successfully identify the flux rope and estimate its scale, location and amplitude.

\section{Application}

In this section, we apply our new method to the spacecraft measurements in the magnetosheath and the magnetotail.

\subsection{Flux rope in the magnetosheath}

Flux ropes are successfully identified in the magnetosheath using the unprecedented high-resolution data from the MMS (Burch et al., 2015) mission (Huang et al., 2016b). Their observations have demonstrated that highly dynamical strong- wave activities and electron-scale physics occur in the magnetosheath ion-scale flux ropes. Figure 6 gives the observations of $\sim 14 \mathrm{~s}$ from MMS2 on 25 October 2015 and the test results of our method. The unit length of the TFC uses the same unit as the real observations, i.e., seconds ("s"). The amplitude $\left(B_{0}\right)$ of the TFC is determined by the maximum value of $B_{\mathrm{t}}$ during the interval when calculating correlation coefficients. Similar to the model test, we use the same variables to present the components of the bipolar variation, core field and total magnetic field after transformed to MVA (Huang et al., 2016b). The threshold of the correlation coefficients is also set as 0.9 in Fig. 6 . We can see that the correlation coefficients of the three variables (Fig. 6b-d) only have high values at the same time around time $=5.5 \mathrm{~s}$, implying that one flux rope is identified by this method. Based on the weighted-average method in Eq. (2), the timescale of the flux rope is $1.11 \mathrm{~s}$, and its central location is at $5.38 \mathrm{~s}$. The amplitude is estimated as $115 \mathrm{nT}$. All these results are consistent with previous findings from multispacecraft data in Huang et al. (2016b).

\subsection{Flux rope in the magnetotail}

Flux ropes are frequently observed in the magnetotail and play an important role during magnetic reconnection and magnetotail dynamics (e.g., Slavin et al., 2003; Zong et al., 2004; Chen et al., 2007; Huang et al., 2012, 2016a; Fu et al., 2015, 2016). Chen et al. (2008) have identified several flux ropes filled with energetic electrons during magnetic recon- 

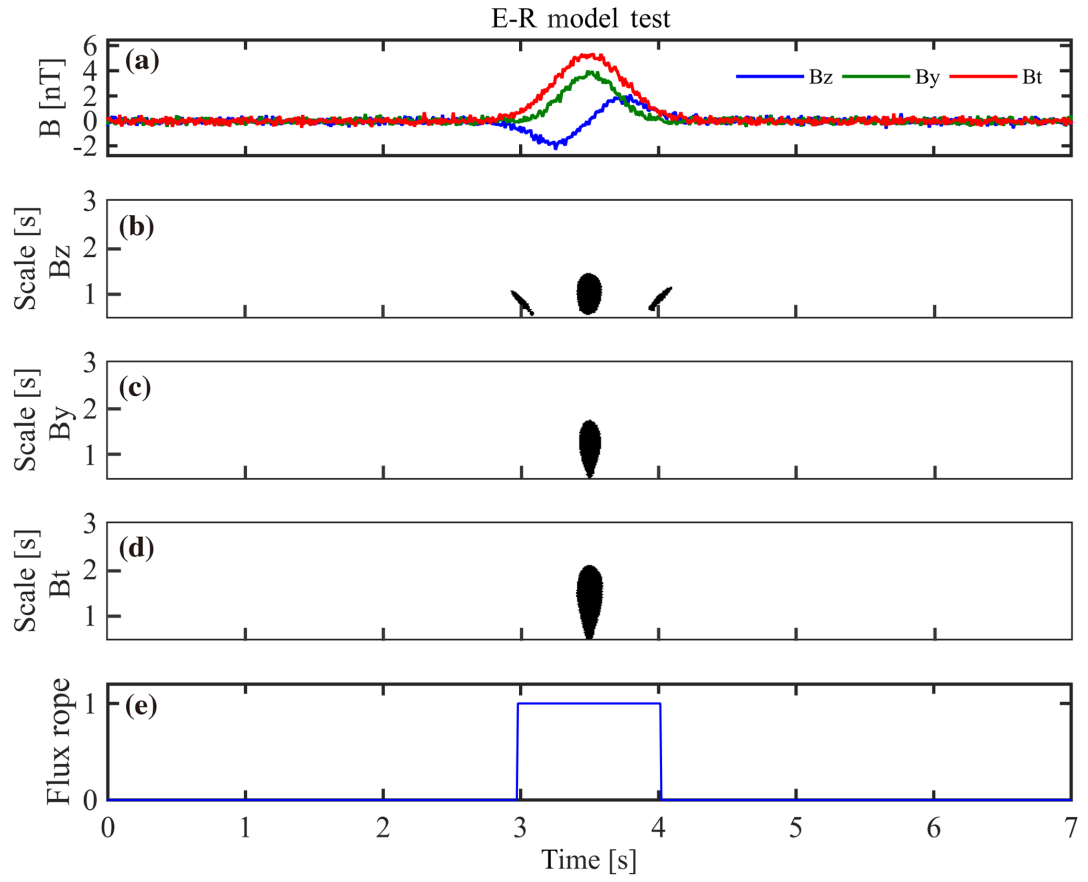

Figure 5. The test results on $E-R$ model with a threshold of 0.9. (a) Three variables $B_{z}, B_{y}$ and $B_{\mathrm{t}}$ from $E-R$ model with $10 \%$ random noise; (b-d) the correlation coefficients ( $\geq 0.9$ ) between the variables of $B_{z}, B_{y}$ and $B_{\mathrm{t}}$ and the TFC, respectively; (e) the index when the virtual spacecraft cross the flux rope (if the spacecraft cross the flux rope, the index is 1 ; if not, the index is 0 ). The duration of the index presents the timescale of the flux rope. The scale on the vertical axes of (b-d) is the same as in Fig. 4.
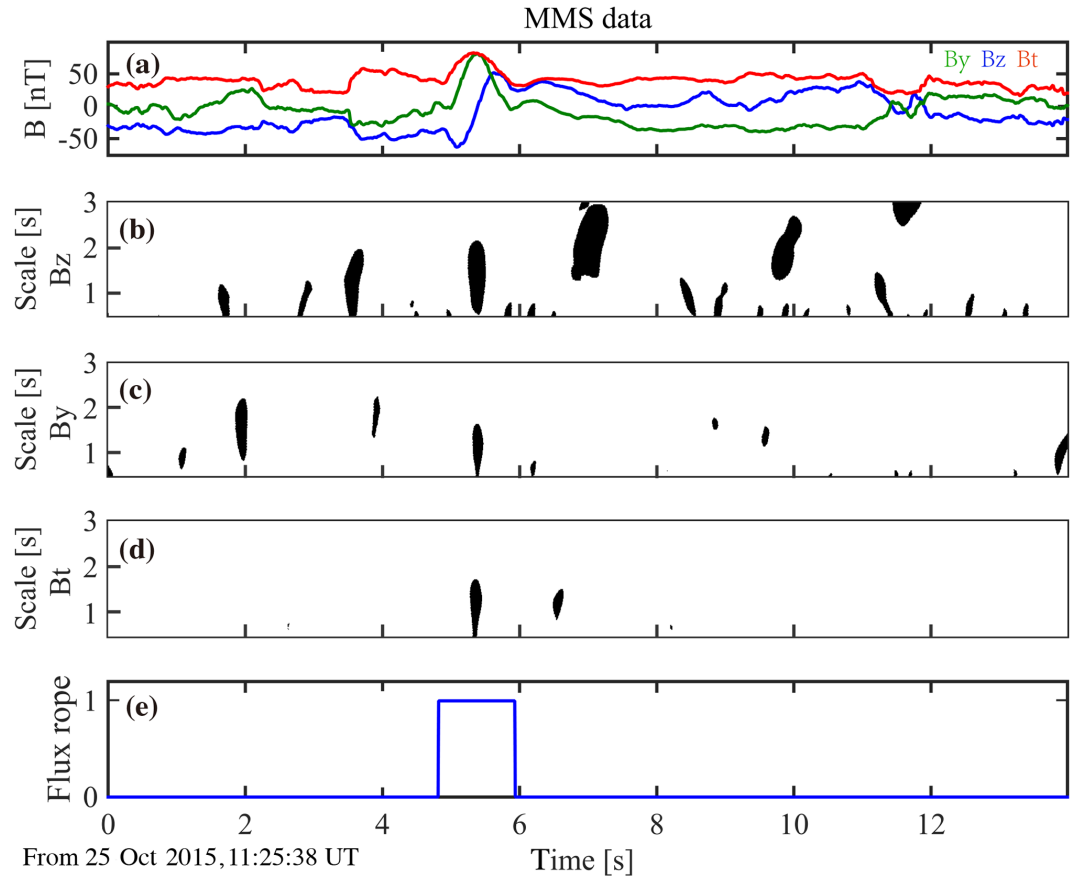

Figure 6. Testing the method on MMS data in the magnetosheath. The same format as in Fig. 5. The scale on the vertical axes of (b-d) uses seconds as the unit. 


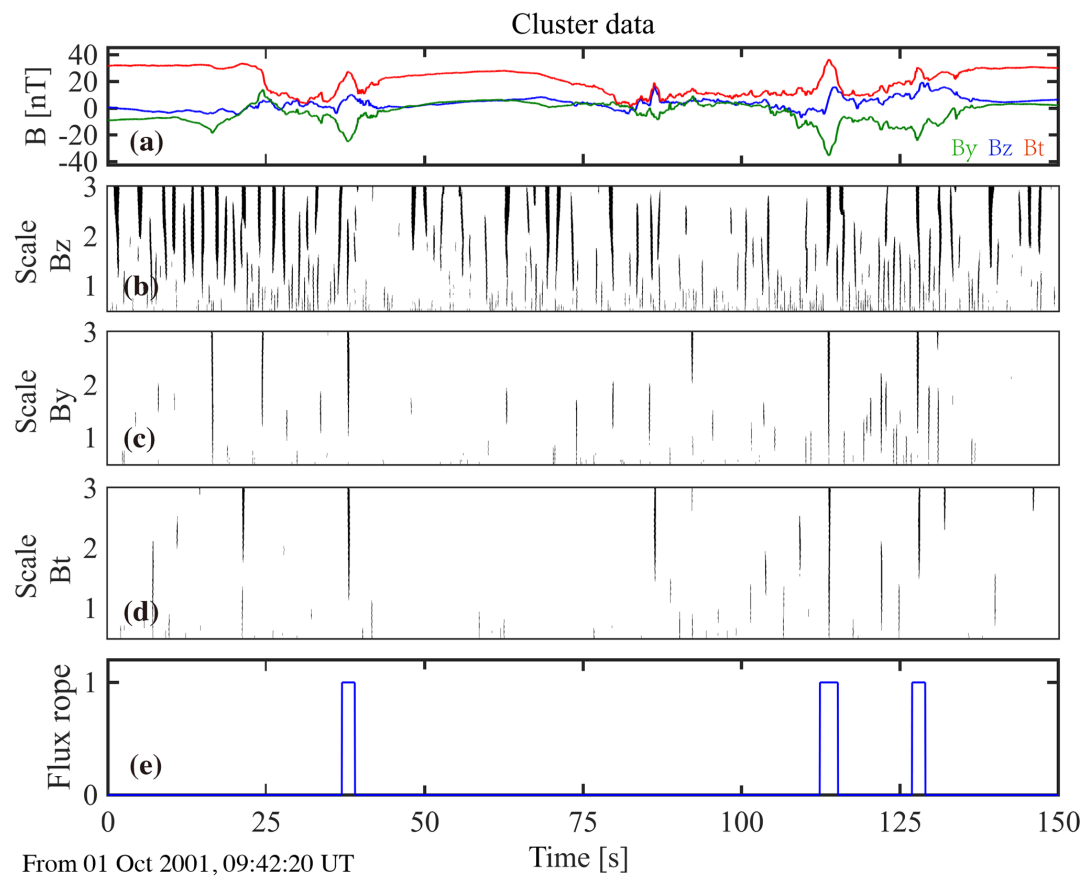

Figure 7. Testing the method on Cluster data in the magnetotail. The same format as in Fig. 6.

Table 1. The location, scale and amplitude of the flux ropes identified by the method. The amplitude is defined as the values of the bipolar variation from minimum to maximum.

\begin{tabular}{lrrr}
\hline No. of flux rope & 1 & 2 & 3 \\
\hline Location (s) & 37.91 & 113.79 & 127.93 \\
Scale (s) & 1.99 & 2.84 & 2.05 \\
Amplitude (nT) & 9.96 & 20.49 & 12.59 \\
\hline
\end{tabular}

nection on 1 October 2001 by using the Cluster data. Figure 7 shows the magnetic field in GSM coordinates from the Cluster mission (Escoubet et al., 1997) in the magnetotail and the application results of our method. There are several bipolar variations in $B_{z}$ during this time interval (Fig. 7a). Figure $7 \mathrm{~b}-\mathrm{d}$ present the correlation coefficients (larger than 0.9 of the threshold) of the three variables. Here we try to identify small-scale flux ropes, so that we perform the method only at short timescales. These are full of high correlation coefficients (grey shadows in Fig. 7b-d). After compare with the correlation coefficients at the same time and same scale, our method resolves three possible flux ropes in Fig. 7e. The results are summarized in Table 1 . The three structures are close to the ideal flux rope with bipolar signature in $B_{z}$, and peaks in core field $B_{y}$ and total magnetic field $B_{\mathrm{t}}$. All three flux ropes identified by our method have been reported in Chen et al. (2007).

We should point out that our method can only identify the flux rope and derive its duration. If the plasma velocity data are available, then we can estimate the actual spatial scale of the flux ropes. If multispacecraft data are available for the time interval of interest, one can derive the size, the orientation and the motion of the flux rope using the multispacecraft methods such as those of Sonnerup et al. (2004), Shi et al. $(2005,2006)$ and Zhou et al. (2006a, b). However, the separation of the Cluster was much larger than the size of the flux ropes on 1 October 2001, implying that one cannot use the multispacecraft method here.

\section{Summary and discussion}

In summary, we developed a new method to identify flux ropes in the space plasmas. This method is based on the correlation coefficients between the signal and the TFC from the non-force-free $E-R$ model. If the correlation coefficients of three variables $\left(B_{z}, B_{y}\right.$ and $\left.B_{\mathrm{t}}\right)$ of the signal have high values of correlation coefficients at the same time and same scale, one can deduce the existence of one flux rope and estimate its location and its timescale (i.e., the duration). The tests on the artificial data and the in situ realistic spacecraft data show that our method can successfully search out the flux ropes and obtain their locations and timescales.

Bipolar variation in the $B_{z}$ component and the enhancement in core field and magnetic field strength are the typical signatures for most flux ropes. But it does not mean that all observations from any crossing of the spacecraft would have those signatures, which depends on the spacecraft trajectory (especially for bipolar components). However, one only can 
select or identify the flux rope showing the typical signatures and miss other flux rope that do not have the typical signatures. Some special field structures may induce similar signatures along some special trajectories. But this opportunity does not often occur in the magnetotail. Moreover, one can use the plasma measurements to rule out this possibility.

The aforementioned attempts are made to identify flux ropes in the Earth's magnetotail by eyes or half-automatically based on the bipolar variation of $B_{z}$ (e.g., Richardson et al., 1987; Slavin et al., 2003; Kawano and Russell, 1996; Vogt et al., 2010; Jackman et al., 2014; Smith et al., 2016). The identifications by eyes would miss a lots of flux ropes and take too much time. Karimabadi et al. (2009) used a data mining technique (MineTool) to search flux ropes using both magnetic field and plasma data. That method is too complex to apply in the data analysis. Smith et al. (2017) proposed one method to automatically detect force-free flux ropes based on magnetic field data from a single spacecraft. In the present study, we used the TFC derived from non-force-free flux rope model to calculate the correlation coefficients with the signal, and then compare the large correlation coefficients of different variables to identify the flux rope. Our method is flexible, reliable and easy to apply with the in situ spacecraft data compared with other methods. We will quantitatively model the flux ropes identified by our method and derive more information on the flux ropes. For example, we can statistically survey and investigate the locations, scales and global distributions of flux ropes in the magnetosheath using MMS data.

We should point out that there are several limitations in our method:

1. Our method can only detect the nearly ideal cylindrical flux rope since we used non-force-free $E-R$ model to describe the TFC, which limits the application of this method. The non-force-free model proposed by $E-R$ is just one possible solution of all the flux rope that satisfies $J \times B \neq 0$. Actually, one can use other flux rope models to replace $E-R$ model and extend our method to identify the flux ropes.

2. If the flux ropes are not regular, there are large time deviations among $B_{z}, B_{y}$ and $B_{\mathrm{t}}$ that will lead to some flux ropes being missed when we apply the method.

3. The threshold value of correlation coefficients can affect the results, such as when the threshold value is so small that the method detects some possible structures that do not belong to flux ropes, or so large that the method will miss some flux ropes.

4. The correlation coefficients at small scales (especially in $B_{y}$ and $B_{\mathrm{t}}$ ) could be very large, which may affect our results. The method may find some possible structures related to such fluctuations. We will improve this method and apply it to detect the flux ropes in the turbulent magnetosheath in the future.
Data availability. MMS Data are publicly available from the MMS Science Data Center at http://lasp.colorado.edu/mms/sdc/ (last access: September 2018). Cluster data are publicly available from the Cluster Science Archive at http://www.cosmos.esa.int/web/csa (last access: July 2018).

Author contributions. SH and JH proposed the algorithm. PZ coded the algorithm and tested and analyzed the algorithm output data. SH also helped with the algorithm development and analyzed the data. RT and JB provided MMS data. SH wrote the paper, and all others commented on it.

Competing interests. The authors declare that they have no conflict of interest.

Acknowledgements. We thank the entire Cluster and MMS team and instrument leads for data access and support. This work was supported by the National Natural Science Foundation of China (41574168, 41674161, 41874191). Shiyong Huang acknowledges the support by Young Elite Scientists Sponsorship Program by CAST (2017QNRC001).

Edited by: Christopher Mouikis

Reviewed by: two anonymous referees

\section{References}

Borg, A. L., Taylor, M. G. G. T., and Eastwood, J. P.: Observations of magnetic flux ropes during magnetic reconnection in the Earth's magnetotail, Ann. Geophys., 30, 761-773, https://doi.org/10.5194/angeo-30-761-2012, 2012.

Burch, J. L., Moore, T. E., Torbert, R. B., and Giles, B. L.: Magnetospheric Multiscale overview and science objectives, Space Sci Rev., 199, 5, https://doi.org/10.1007/s11214-015-0164-9, 2015.

Chen, L.-J., Bhattacharjee, A., Puhl-Quinn, P. A., Yang, H., Bessho, N., Imada, S., Mühlbachler, S., Daly, P. W., Lefebvre, B., Khotyaintsev, Y., Vaivads, A., Fazakerley, A., and Georgescu, E.: Observation of energetic electrons within magnetic islands, Nature Phys., 4, 19-23, https://doi.org/10.1038/nphys777, 2007.

Daughton, W., Scudder, J., and Karimabadi, H.: Fully kinetic simulations of undriven magnetic reconnection with open boundary conditions, Phys. Plasmas, 13, 072101, https://doi.org/10.1063/1.2218817, 2006.

Deng, X. H., Matsumoto, H., Kojima, H., Mukai, T., Anderson, R. R., Baumjohann, W., and Nakamura, R.: Geotail encounter with reconnection diffusion region in the Earth's magnetotail: Evidence of multiple $\mathrm{X}$ lines collisionless reconnection?, J. Geophys. Res., 109, A05206, https://doi.org/10.1029/2003JA010031, 2004.

Drake, J. F., Swisdak, M., Che, H., and Shay, M. A.: Electron acceleration from contracting magnetic islands during reconnection, Nature, 443, 553-556, 2006.

Elphic, R. C. and Russell, C. T.: Magnetic Flux Ropes in the Venus Ionosphere: Observations and Models, J. Geophys. Res., 88, 5872, https://doi.org/10.1029/JA088iA01p00058, 1983. 
Escoubet, C. P., Schmidt, R., and Goldstein, M. L.: Cluster-Science and mission overview, Space Sci. Rev., 79, 11-32, 1997.

Fu, H. S., Cao, J. B., Khotyaintsev, Yu. V., Sitnov, M. I., Runov, A., Fu, S. Y., Hamrin, M., André, M., Retinò, A., Ma, Y. D., Lu, H. Y., Wei, X. H., and Huang, S. Y.: Dipolarization fronts as a consequence of transient reconnection: In situ evidence, Geophys. Res. Lett., 40, 6023-6027, https://doi.org/10.1002/2013GL058620, 2013.

Fu, H. S., Vaivads, A., Khotyaintsev, Y. V., Olshevsky, V., André, M., Cao, J. B., Huang, S. Y., Retinò, A., and Lapenta, G.: How to find magnetic nulls and reconstruct field topology with MMS data?, J. Geophys. Res.-Space Phys., 120, 3758-3782, https://doi.org/10.1002/2015JA021082, 2015.

Fu, H. S., Cao, J. B., Vaivads, A., Khotyaintsev, Y. V., Andre, M., Dunlop, M., Liu, W. L., Lu, H. Y., Huang, S. Y., Ma, Y. D., and Eriksson, E.: Identifying magnetic reconnection events using the FOTE method, J. Geophys. Res.-Space Phys., 121, 1263-1272, https://doi.org/10.1002/2015JA021701, 2016.

Fu, H. S., Vaivads, A., Khotyaintsev, Y. V., André, M., Cao, J. B., Olshevsky, V., Eastwood, J. P., and Retinò, A.: Intermittent energy dissipation by turbulent reconnection, Geophys. Res. Lett., 44, 37-43, https://doi.org/10.1002/2016GL071787, 2017.

Hidalgo, M. A., Cid, C., Vinas, A. F., and Sequeiros, J.: A non-force-free approach to the topology of magnetic clouds in the solar wind, J. Geophys. Res., 106, 1002, https://doi.org/10.1029/2001JA900100, 2002.

Hotelling, H.: New Light on the Correlation Coefficient and its Transforms, J. Roy. Stat. Soc. B, 15, 193-232, 1953.

$\mathrm{Hu}$, Q. and Sonnerup, B. U. O.: Reconstruction of magnetic flux ropes in the solar wind, Geophys. Res. Lett., 28, 467-470, 2001.

$\mathrm{Hu}, \mathrm{Q}$. and Sonnerup, B. U. O.: Reconstruction of magnetic clouds in the solar wind: Orientations and configurations, J. Geophys. Res., 107, 1142, https://doi.org/10.1029/2001JA000293, 2002.

Huang, S. Y., Vaivads, A., Khotyaintsev, Y. V., Zhou, M., Fu, H. S., Retinò, A., Deng, X. H., André, M., Cully, C. M., He, J. S., Sahraoui, F., Yuan, Z. G., and Pang, Y.: Electron acceleration in the reconnection diffusion region: Cluster observations, Geophys. Res. Lett., 39, L11103, https://doi.org/10.1029/2012GL051946, 2012.

Huang, S. Y., Pang, Y., Yuan, Z., Deng, X., He, J., Zhou, M., Fu, H., Fu, S., Li, H., Wang, D., and Li, H.: Observation of directional change of core field inside flux ropes within one reconnection diffusion region in the Earth's magnetotail, Chin. Sci. Bull., 59, 4797-4803, https://doi.org/10.1007/s11434-014-0583-0, 2014a.

Huang, S. Y., Zhou, M., Yuan, Z. G., Deng, X. H., Sahraoui, F., Pang, Y., and Fu, S.: Kinetic simulations of electric field structure within magnetic island during magnetic reconnection and their applications to the satellite observations, J. Geophys. Res.-Space Phys., 119, 7402-7412, https://doi.org/10.1002/2014JA020054, 2014b.

Huang, S. Y., Zhou, M., Yuan, Z. G., Fu, H. S., He, J. S., Sahraoui, F., Aunai, N., Deng, X. H., Fu, S., Pang, Y., and Wang, D. D.: Kinetic simulations of secondary reconnection in the reconnection jet, J. Geophys. Res.-Space Phys., 120, 6188-6198, https://doi.org/10.1002/2014JA020969, 2015.

Huang, S. Y., Retino, A., Phan, T. D., Daughton, W., Vaivads, A., Karimabadi, H., Zhou, M., Sahraoui, F., Li, G. L., Yuan, Z. G., Deng, X. H., Fu, H. S., Fu, S., Pang, Y., and Wang, D. D.: In situ observations of flux rope at the separatrix region of mag- netic reconnection, J. Geophys. Res.-Space Phys., 121, 205-213, https://doi.org/10.1002/2015JA021468, 2016a.

Huang, S. Y., Sahraoui, F., Retino, A., Le Contel, O., Yuan, Z. G., Chasapis, A., Aunai, N., Breuillard, H., Deng, X. H., Zhou, M., Fu, H. S., Pang, Y., Wang, D. D., Torbert, R. B., Goodrich, K. A., Ergun, R. E., Khotyaintsev, Y. V., Lindqvist, P.-A., Russell, C. T., Strangeway, R. J., Magnes, W., Bromund, K., Leinweber, H., Plaschke, F., Anderson, B. J., Pollock, C. J., Giles, B. L., Moore, T. E., and Burch, J. L.: MMS observations of ion-scale magnetic island in the magnetosheath turbulent plasma, Geophys. Res. Lett., 43, 7850-7858, https://doi.org/10.1002/2016GL070033, 2016b.

Hughes, W. J., Sibeck, D. G.: On the 3-dimensional structure of plasmoids, Geophys. Res. Lett., 14, 636-639, 2013.

Jackman, C. M., Slavin, J. A., Kivelson, M. G., Southwood, D. J., Achilleos, N., Thomsen, M. F., DiBraccio, G. A., Eastwood, J. P., Freeman, M. P., Dougherty, M. K., and Vogt, M. F.: Saturn's dynamic magnetotail: A comprehensive magnetic field and plasma survey of plasmoids and traveling compression regions and their role in global magnetospheric dynamics, J. Geophys. Res.-Space Phys., 119, 5465-5494, https://doi.org/10.1002/2013JA019388, 2014.

Karimabadi, H., Sipes, T. B., Wang, Y., Lavraud, B., and Roberts, A.: A new multivariate time series data analysis technique: Automated detection of flux transfer events using Cluster data, J. Geophys. Res., 114, A06216, https://doi.org/10.1029/2009JA014202, 2009.

Kawano, H. and Russell, C. T.: Survey of flux transfer events observed with the ISEE 1 spacecraft: Rotational polarity and the source region, J. Geophys. Res., 101, 27299-27308, 1996.

Lee, L. C., Fu, Z. F., and Akasofu, S.-I.: A simulation study of forced reconnection processes and magnetospheric storms and substorms, J. Geophys. Res., 90, 10896-10910, 1985.

Lepping, R. P., Jones, J. A., and Burlaga, L. F.: Magnetic field structure of interplanetary magnetic clouds at 1 AU, J. Geophys. Res., 95, 11957-11965, 1990.

Nakamura, M. and Scholer, M.: Structure of the magnetopause reconnection layer and of flux transfer events: Ion kinetic effects, J. Geophys. Res., 105, 23179-23191, https://doi.org/10.1029/2000JA900101, 2000.

Richardson, I. G., Cowley, S. W. H., Hones, E. W., and Bame, S. J.: Plasmoid-associated energetic ion bursts in the deep geomagnetic tail: Properties of plasmoids and the postplasmoid plasma sheet, J. Geophys. Res., 2, 9997-10013, https://doi.org/10.1029/JA092iA09p09997, 1987.

Rong, Z. J., Wan, W. X., Shen, C., Zhang, T. L., Lui, A. T. Y., Wang, Y., Dunlop, M. W., Zhang, Y. C., and Zong, Q.-G.: Method for inferring the axis orientation of cylindrical magnetic flux rope based on single-point measurement, J. Geophys. Res.-Space Phys., 118, 271-283, https://doi.org/10.1029/2012JA018079, 2013.

Slavin, J. A., Lepping, R. P., Gjerloev, J., Fairfield, D. H., Hesse, M., Owen, C. J., Moldwin, M. B., Nagai, T., Ieda, A., and Mukai, T.: Geotail observations of magnetic flux ropes in the plasma sheet, J. Geophys. Res., 108, 1015-1032, 2003.

Shi, Q. Q., Shen, C., Pu, Z. Y., Dunlop, M. W., Zong, Q.-G., Zhang, H., Xiao, C. J., Liu, Z. X., and Balogh, A.: Dimensional analysis of observed structures using multipoint magnetic field measure- 
ments: Application to Cluster, Geophys. Res. Lett., 32, L12105, https://doi.org/10.1029/2005GL022454, 2005.

Shi, Q. Q., Shen, C., Dunlop, M. W., Pu, Z. Y., Zong, Q.-G., Liu, Z.-X., Lucek, E. A., and Balogh, A.: Motion of observed structures calculated from multi-point magnetic field measurements: Application to Cluster, Geophys. Res. Lett., 33, L08109, https://doi.org/10.1029/2005GL025073, 2006.

Smith, A. W., Jackman, C. M., and Thomsen, M. F.: Magnetic reconnection in Saturn's magnetotail: A comprehensive magnetic field survey, J. Geophys. Res.-Space Phys., 121, 2984-3005, https://doi.org/10.1002/2015JA022005, 2016.

Smith, A. W., Slavin, J. A., Jackman, C. M., Fear, R. C., Poh, G.-K., DiBraccio, G. A., Jasinski, J. M., and Trenchi, L. (2017), Automated force free flux rope identification,J. Geophys. Res.-Space Phys., 122, 780-791, https://doi.org/10.1002/2016JA022994, 2017.

Sonnerup, B. U. O., Hasegawa, H., and Paschmann, G.: Anatomy of a flux transfer event seen by Cluster, Geophys. Res. Lett., 31, L11803, https://doi.org/10.1029/2004GL020134, 2004.

Vogt, M. F., Kivelson, M. G., Khurana, K. K., Joy, S. P., and Walker, R. J.: Reconnection and flows in the Jovian magnetotail as inferred from magnetometer observations, J. Geophys. Res., 115, A06219, https://doi.org/10.1029/2009JA015098, 2010.

Wang, R., Lu, Q., Du, A., and Wang, S.: In Situ Observations of a Secondary Magnetic Island in an Ion Diffusion Region and Associated Energetic Electrons, Phys. Rev. Lett., 104, 175003, 2010.
Wang, R., Lu, Q., Nakamura, R., Huang, C., Du, A., Guo, F., Teh, W., Wu, M., Lu, S., and Wang, S.: Coalescence of magnetic flux ropes in the ion diffusion region of magnetic reconnection, Nature Phys., 12, 263-267, https://doi.org/10.1038/nphys3578, 2016.

Zhang, H., Kivelson, M. G., Khurana, K. K., McFadden, J., Walker, R. J., Angelopoulos, V., Weygand, J. M., Phan, T., Larson, D., Glassmeier, K. H., and Auster, H. U.: Evidence that crater flux transfer events are initial stages of typical flux transfer events, J. Geophys. Res., 115, A08229, https://doi.org/10.1029/2009JA015013, 2010.

Zhou, X.-Z., Zong, Q.-G., Pu, Z. Y., Fritz, T. A., Dunlop, M. W., Shi, Q. Q., Wang, J., and Wei, Y.: Multiple Triangulation Analysis: another approach to determine the orientation of magnetic flux ropes, Ann. Geophys., 24, 1759-1765, https://doi.org/10.5194/angeo-24-1759-2006, 2006a.

Zhou, X.-Z., Zong, Q.-G., Wang, J., Pu, Z. Y., Zhang, X. G., Shi, Q. Q., and Cao, J. B.: Multiple triangulation analysis: application to determine the velocity of 2-D structures, Ann. Geophys., 24, 3173-3177, https://doi.org/10.5194/angeo-24-31732006, 2006b.

Zong, Q.-G., Fritz, T. A., Pu, Z. Y., Fu, S. Y., Baker, D. N., Zhang, H., Lui, A. T., Vogiatzis, I., Glassmeier, K.-H., Korth, A., Daly, P. W., Balogh, A., and Reme, H.: Cluster observations of earthward flowing magnetic island in the tail, Geophys. Res. Lett., 31, L18803, https://doi.org/10.1029/2004GL020692, 2004. 